\title{
Photoluminescence Properties of $\mathrm{LaF}_{3}-$ Coated Porous Silicon
}

\author{
Sinthia Shabnam Mou, Md. Johurul Islam, Abu Bakar Md. Ismail* \\ Department of Applied Physics and Electronic Engineering, Rajshahi University, Rajshahi, Banglaesh. \\ Email: ismail@ru.ac.bd
}

Received November $12^{\text {th }}, 2010$; revised January $8^{\text {th }}, 2011$; accepted May $17^{\text {th }}, 2011$.

\begin{abstract}
This work reports the coating of porous silicon (PS) with $\mathrm{LaF}_{3}$ and its influence on the photoluminescence (PL) property of PS. PS samples, prepared by electrochemical etching in a solution of HF and ethanol, were coated with e-beam evaporated-LaF ${ }_{3}$ of different thicknesses. It was observed that the thin $L_{a F} F_{3}$ layer on PS led to a good enhancement of $P L$ yield of PS. But with the increasing thickness of LaF ${ }_{3}$ layer PL intensity of PS was decreasing along with a small blue-shift. It was also observed that all the coated samples showed degradation in PL intensity with time, but annealing could recover and stabilize the degraded PL.
\end{abstract}

Keywords: Porous Silicon, Anodic Etching, Photoluminescence, Surface Coating, Photonics

\section{Introduction}

Porous silicon (PS) can be considered as a silicon (Si) crystal having a network of voids in it [1]. The nanosized voids in the Si bulk result in a sponge-like structure of pores and channels surrounded with a skeleton of crystalline Si nanowires. Because of its versatility and tunable characteristics, PS has attracted much attention in optoelectronic industry due to its considerably strong photoluminescence (PL) behavior at room temperature apart from its various applications such as sensor and MEMS. As it has a potential to emit visible light, it is expected that this material can be effectively used to fabricate Si-based visible light emitting devices and optical interconnections. The PL phenomena of PS were reported in the red light region first, while orange, yellow and green PL was also observed latter. The wide spectral tunability of the PL of PS that extends from near infrared (IR) through the whole visible range to the near ultraviolet (UV) make this material a very interesting one. A disadvantage of this material is the aging, that is, the slow spontaneous oxidation of PS [2]. A native oxide layer forms on the surface of the pores and the thickness of this oxide layer grows with time. Due to the aging effect, the structural and optical properties of PS show continuous change with storage time [2]. That is, many of its properties, such as PL, are age dependent and unstable, and why PS is still can not be applied in photonics. One possible way to reduce the aging effect could be "passiva- tion" of PS [3]. This surface coating can be achieved from various chemical adsorbates by several techniques [4-6].

In this work, an attempt has been made to coat PS surface by Lanthanum Fluoride $\left(\mathrm{LaF}_{3}\right)$, aiming to obtain stable and enhanced luminescence of PS layers. $\mathrm{LaF}_{3}$ is a large band-gap (about $10.3 \mathrm{eV}$ ) [7] material having a hexagonal crystal structure with a refractive index of 1.61 [8]. Lanthanum fluoride is a promising vacuum ultraviolet (VUV) transparent material similar to the other large band-gap fluorides such as $\mathrm{GdF}_{3}$ and $\mathrm{MgF}_{2}$. Due to its high band-gap and refractive index, higher than those of the other VUV transparent films, $\mathrm{LaF}_{3}$ is a useful material for VUV optics. Therefore, the PL properties of anodically etched PS with and without $\mathrm{LaF}_{3}$-coating have been compared and the influence of $\mathrm{LaF}_{3}$-coating on the PL has been discussed in this article. The experimental results show that coating the PS with optimum $\mathrm{LaF}_{3}$ thickness could provide stabilization of PL of PS, which is very important for PS to be a potential material in photonic devices.

\section{Experimental}

PS was prepared by anodic etching of $\mathrm{p}^{+}$-type single crystal silicon wafer having $<100>$ orientation, $10-24 \Omega$ $\mathrm{cm}$ resistivity and $200 \pm 20 \mu \mathrm{m}$ thickness. Etching was done in a $3: 1(\mathrm{v} / \mathrm{v})$ solution of $48 \% \mathrm{HF}$ and absolute ethanol. PS samples with various current densities and 
etching times were prepared. Among them, the samples with $15 \mathrm{~mA} / \mathrm{cm}^{2}$ current density and 10 min etching time were coated with $\mathrm{LaF}_{3}$ of three different thicknesses $(70$ $\pm 10 \mathrm{~nm}, 120 \pm 10 \mathrm{~nm}$ and $180 \pm 10 \mathrm{~nm}$ ). The etching or electrochemical dissolution of $\mathrm{Si}$ wafer was done using single tank cell arrangement, where the Si itself was used as the anode and a platinum (Pt) electrode was used as the cathode. The back contact of the Si wafer was done by depositing a thick silver (Ag) layer by electron-beam (e-beam) evaporation. The anhydrous $\mathrm{LaF}_{3}$ with $99.999 \%$ purity was bought from the Kishida Co., Japan, and was used without further purification. $\mathrm{LaF}_{3}$ layers were deposited by e-beam evaporation in a vacuum coating unit. A high vacuum of $10^{-6}$ Torr was maintained during evaporation of $\mathrm{LaF}_{3}$. PL study was done by Spectro Fluorophotometer where the excitation wavelength was $350 \mathrm{~nm}$.

Just after anodic etching the PS samples were dried and put inside the vacuum coating unit for $\mathrm{LaF}_{3}$ deposition.

\section{Results and Analysis}

The fabricated PS samples with $\mathrm{LaF}_{3}$ layers were investigated with a scanning electron microscope (SEM). Figures 1 and $\mathbf{2}$ show the SEM image of the surface and cross-section of a $\mathrm{LaF}_{3}$-coated PS sample with a $180 \pm 10$ $\mathrm{nm} \mathrm{LaF} 3$ layer. Since $\mathrm{LaF}_{3}$ covers the whole surface the pore tips are not visible. The $\mathrm{LaF}_{3} / \mathrm{PS} / \mathrm{Si}$ heterostructure (from left to right in the image) is clearly visible in Figure 2. For both sets of the as-grown PS sample, single PL peak appeared around $695 \mathrm{~nm}$, which is similar to the reported value $[9,10]$. The oxygen content of the film was very low which is evident from the single-peak PL band of PS [11]. The PL spectra of PS coated with $\mathrm{LaF}_{3}$ layer of three different thicknesses with an uncoated PS sample has been compared and shown in Figure 3.

It is clearly seen from the figure that coating PS with thin (e.g. $70 \mathrm{~nm}$ ) $\mathrm{LaF}_{3}$ layer could protect the PS from its PL intensity-degradation, which may be related to the coating effect of the $\mathrm{LaF}_{3}$ that prevents the PS surface chemistry. But with the increase in the thickness of $\mathrm{LaF}_{3}$, the PL intensity is decreased. And for the sample with $\mathrm{LaF}_{3}$ of $180 \pm 10 \mathrm{~nm}$ thickness, the PL intensity of PS is decreased even below the PL intensity of the uncoated PS. The decrease in the intensity of the PL emission with increasing thickness of $\mathrm{LaF}_{3}$ can be explained by the absorption of light by the $\mathrm{LaF}_{3}$ shell. The light emitted by the PS is absorbed by the $\mathrm{LaF}_{3}$ when it passes through the $\mathrm{LaF}_{3}$ layer. The absorption is proportional to the thickness of the $\mathrm{LaF}_{3}$ layer. Therefore, the intensity of the PL emission decreases with the increase of the $\mathrm{LaF}_{3}$ thickness. The important thing is the shape of the PL response that did not change due to coating with $\mathrm{LaF}_{3}$.

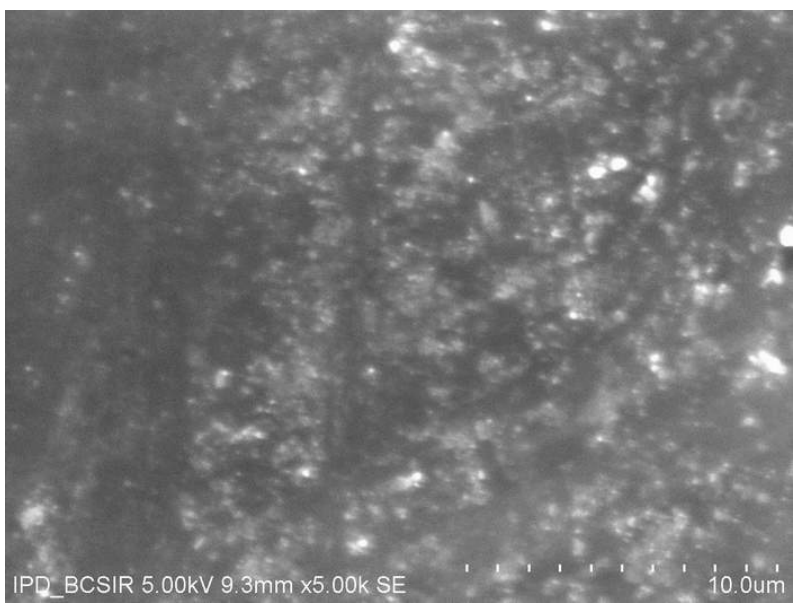

Figure 1. SEM image of $\mathrm{LaF}_{3}$-coated PS surface.

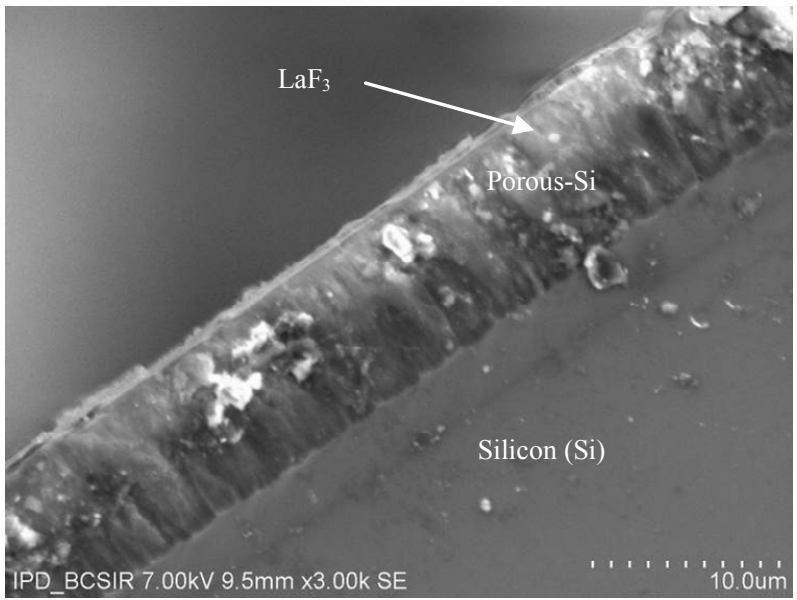

Figure 2. Cross-sectional SEM image of PS sample coated with $\mathrm{LaF}_{3}$.

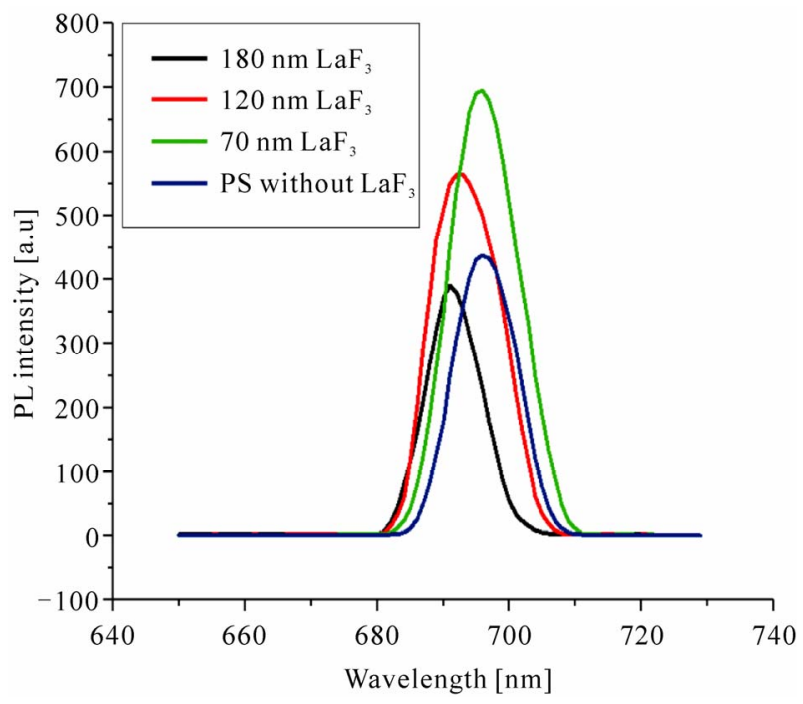

Figure 3. Comparative PL spectra of PS with and without $\mathrm{LaF}_{3}$ coating. 
The full width at half maxima (FWHM) of the Gussian shaped PL response of the coated-PS remained almost constant at $\sim 12 \mathrm{~nm}$. This signifies that the $\mathrm{LaF}_{3}$ layer behaves just as a capping layer that keeps the $\mathrm{Pb}$-center of PS and do not interfere with the PS surface chemistry inside the pore.

The peak wavelengths of the PL spectra of the samples shifted a little towards blue with the increasing thickness of $\mathrm{LaF}_{3}$. This nature of blue-shift has also been reported by Gokarna et al. [12] for the PS/CdS and $\mathrm{ZnS}$ system. This shift in the wave length of the PL can be attributed to the shrinking of nanocrystallites due to thicker $\mathrm{LaF}_{3}$ layer [13]. The shift has been shown in Figure 4. The FWHM of the PL responses of the $\mathrm{LaF}_{3}$-coated PS was very narrow $(\sim 12 \mathrm{~nm})$ that implies the homogeneity of the nanocrystal.

$\mathrm{LaF}_{3}$-coated PS samples were then stored in air for one month and the aging effect on the PL intensity was observed. It was observed that the PL intensity of $\mathrm{LaF}_{3}$ coated PS also decreased due to the aging effect (Figure 5). But all the three samples did not show same amount of degradation in PL intensity. PS sample coated with $\mathrm{LaF}_{3}$ of less thickness $(70 \pm 10 \mathrm{~nm})$ suffered more from aging effect than those coated with thicker $\mathrm{LaF}_{3}$, i.e. the lower the thickness of $\mathrm{LaF}_{3}$, the more was the aging effect.

It appeared from the SEM that the $\mathrm{LaF}_{3}$ layer only deposited as a capping layer and did not deposit into the pore of the porous silicon to passivate the silicon skeleton. The aging effect of the $\mathrm{LaF}_{3}$-coated PS may be due to the trapped oxygen in the pore of the porous silicon that slowly oxidizes the silicon skeleton. As-deposited $\mathrm{LaF}_{3}$ layer shows non-stoichiometry [14] and reach in pores [15]. The thicker layer of the $\mathrm{LaF}_{3}$ could provide protection from the further oxidation of the silicon skeleton from the ambient oxygen, but the thinner layer failed because of pores in the $\mathrm{LaF}_{3}$. That is why the degradation in the PL intensity was more for the thinner layer of as-deposited $\mathrm{LaF}_{3}$ on PS.

But these pores can be removed, and stoichiometry of $\mathrm{LaF}_{3}$ layer can be partially recovered by annealing that may cause the PL stabilization of PS. The degradation of PL and recovery of PL has been compared in the Figure 6. As shown in Figure 6, annealing recovers the PL intensities for all the coated samples. The influence of annealing on the PL of $\mathrm{LaF}_{3}$-coated PS will be reported elsewhere.

\section{Discussion}

Coating of PS with e-beam evaporated $\mathrm{LaF}_{3}$ has been investigated for the first time in order to see the role of $\mathrm{LaF}_{3}$ as a coating layer on the optical properties of PS such as, in enhancing and stabilizing the PL of PS. The

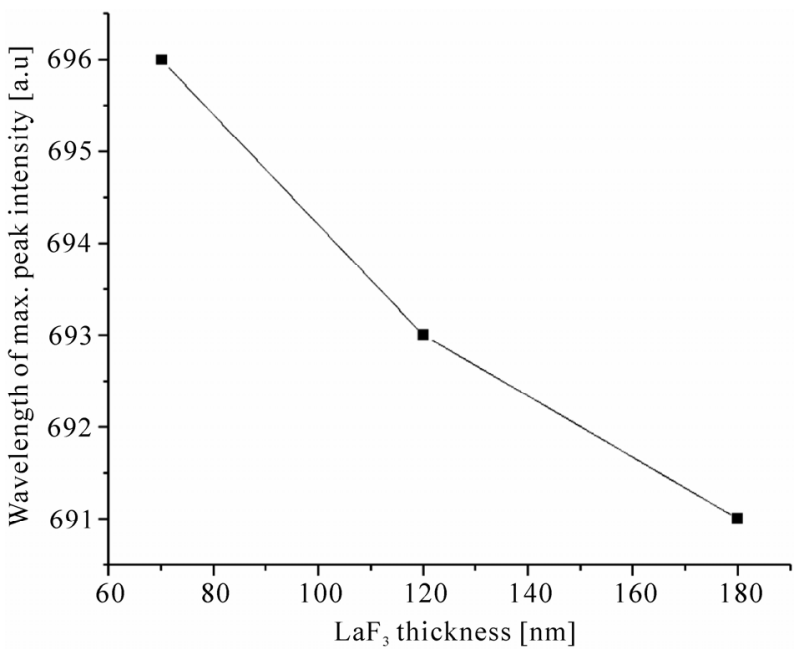

Figure 4. Small blue-shifts with the $\mathrm{LaF}_{3}$ thickness.

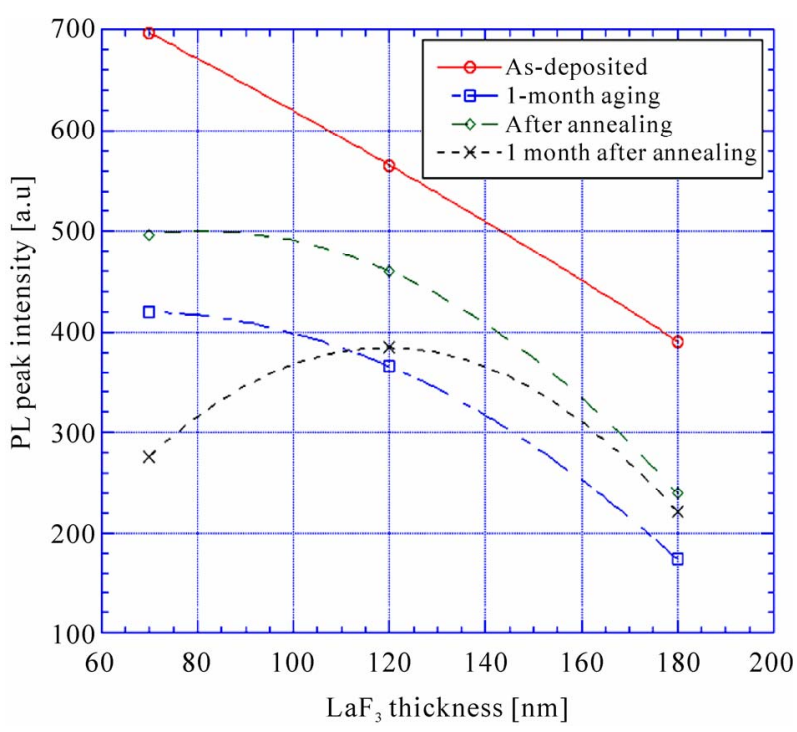

Figure 5. Aging of PL intensity of $\mathrm{LaF}_{3}$-coated PS with time.

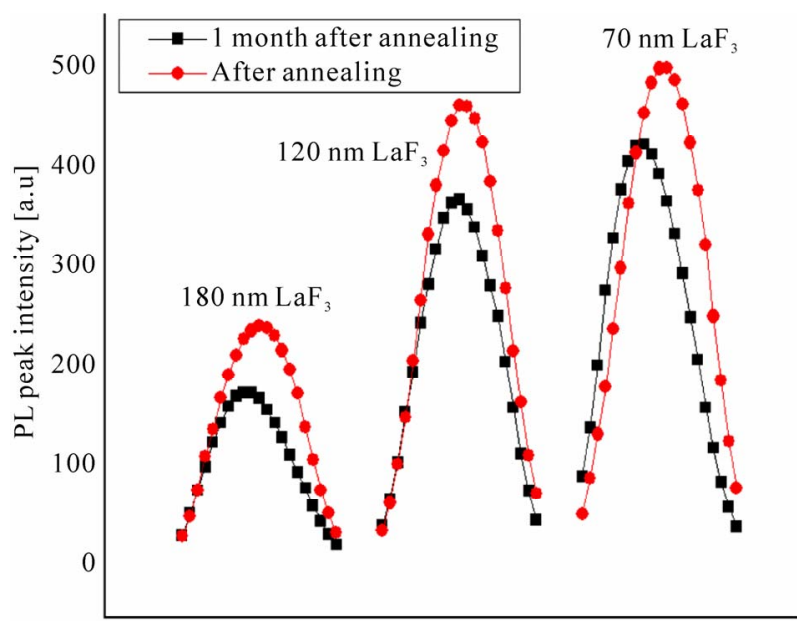

Figure 6. Partial recovery of PL intensity by annealing. 
thickness of the $\mathrm{LaF}_{3}$ appeared to have an important influence on the PL characteristics of PS. Thinner layer of $\mathrm{LaF}_{3}$ could enhance the PL intensity without destroying the Gaussian shape of the PL response. This enhancement of PL is believed to be due to the coating effect of $\mathrm{LaF}_{3}$ that prevented oxidation of PS. But thicker layer of $\mathrm{LaF}_{3}$ lowered the PL intensity that may be due to the degradation of refractive index of the $\mathrm{LaF}_{3}$ layer induced by the mechanical stress which depends on the film thickness. The $\mathrm{LaF}_{3}$-coated un-annealed samples showed aging on the PL intensity. Generally the as-deposited evaporated $\mathrm{LaF}_{3}$ layer contains pores which when exposed to the air water vapor enters into the pore due to capillarity. This water vapor replaces the $\mathrm{Si}-\mathrm{H}$ bonds with Si-O bond which seems responsible for the degradation of the PL intensity. As the thicker layer of as-deposited $\mathrm{LaF}_{3}$ contains more water vapor the degradation of PL intensity was more. When the coated samples were annealed at $400^{\circ} \mathrm{C}$ for 10 minutes the PL intensity was partially recovered and the degradation of the recovered PL intensity was minimum for PS cited with thicker $\mathrm{LaF}_{3}$ layer. Experimental results suggest that coating PS with e-beam evaporated $\mathrm{LaF}_{3}$ and subsequent annealing could enable the PS to have an enhanced and stable PL.

\section{Conclusions}

All the studies done in this work can be summarized as; coating PS with $\mathrm{LaF}_{3}$ of optimum thickness followed by annealing could lead to better luminous stability of PS. From these experimental results it can be concluded that coating with $\mathrm{LaF}_{3}$ of optimum thickness could provide the PS an enhanced and stable photoluminescence that will enable its use in the fabrication of optical devices, such as light-emitting diodes (LED), lasers and solar cells.

\section{Acknowledgements}

The authors wish to thank the COMSTEC, TWAS, Trieste, Italy, the Bangladesh University Grants Commission, and the Faculty of Science of Rajshahi University for providing financial assistance.

\section{REFERENCES}

[1] A. G. Cullis, L. T. Canham and P. D. J. Calcott, "The Structural and Luminescence Properties of Porous Silicon," Journal of Applied Physics, Vol. 82, No. 3, 1997, pp. 909-965. doi:10.1063/1.366536

[2] R. Boukherroub, S. Morin, D. D. M. Wayner and D. J. Lockwood, "Thermal Route for Chemical Modification and Photoluminescence Stabilization of Porous Silicon," Physical Status Solidi (A), Vol. 182, No. 1, 2000, pp 117121.
doi:10.1002/1521-396X(200011)182:1<117::AID-PSSA1 17>3.0.CO;2-3

[3] B. Gelloz, H. Sano, R. Boukherroub, D. D. M. Wayner, D. J. Lockwood and N. Koshida, "Stabilization of Porous Silicon Electroluminescence by Surface Passivation with Controlled Covalent Bonds," Applied Physics Letters, Vol. 83, No. 12, 2003, pp. 2342-2344. doi:10.1063/1.1613812

[4] R. Prabakaran, M. Peres, T. Monteiro, E. Fortunato, R. Martins and I. Ferreira, "The Effects of $\mathrm{ZnO}$ Coating on the Photoluminescence Properties of Porous Silicon for the Advanced Optoelectronic Devices," Journal of Non-Crystalline Solids, Vol. 354, No. 19-25, 2008, pp. 2181-2185.

[5] M. Rahmani, A. Moadhen, M.-A. Zaïbi, H. Elhouichet and M. Oueslati, "Photoluminescence Enhancement and Stabilisation of Porous Silicon Passivated by Iron," Journal of Luminescence, Vol. 128, No. 11, 2008, pp. 1763-1766. doi:10.1016/j.jlumin.2008.04.003

[6] A. G. Macedo, E. A. De Vasconcelos, R. Valaski, F. Muchenski, E. F. Da Silva Jr., A. F. Da Silva and L. S. Roman, "Enhanced Lifetime in Porous Silicon LightEmitting Diodes with Fluorine Doped Tin Oxide Electrodes," Thin Solid Films, Vol. 517, No. 2, 2008, pp. 870873. doi:10.1016/j.tsf.2008.07.007

[7] E. D. Thoma, H. Shields, Y. Zhang, B. C. McCollum, R. T. Williams and J. Lumin, "EPR and Luminescence Studies of $\mathrm{LaF}_{3}$ and $\mathrm{CeF}_{3}$ under X-Ray and Laser Irradiation," Journal of Luminescence, Vol. 71, No. 2, 1997, pp. 93-104. doi:10.1016/S0022-2313(97)81621-3

[8] D. R. Lide, "Physical Constants of Organic Compounds, in: CRC Handbook of Chemistry and Physics," 87th Edition, Taylor and Francis, Boca Raton, 2007.

[9] H. J. Li, J. C. Peng, S. Qu, Y. H. Yan, X. M. Xu and C. J. Zhao, "Efficient Visible Porous Silicon Diodes Passivated by Carbon Films," Chinese Physics Letters, Vol. 19, No. 7, 2002, pp. 1013-1015. doi:10.1088/0256-307X/19/7/341

[10] D. A. Kim, J.-H. Shim and N.-H. Cho, "PL and EL Features of P-Type Porous Silicon Prepared by Electrochemical Anodic Etching," Applied Surface Science, Vol. 234, No. 1-4, 2004, pp. 256-261.

doi:10.1016/j.apsusc.2004.05.028

[11] M. Ohmukai, M. Taniguchi and Y. Tsutsumi, "Large current Density and Anodization Time Needed for Strong Photoluminescence in Porous Silicon," Materials Science and Engineering B, Vol. 86, No. 1, 2001, pp. 26-29. doi:10.1016/S0921-5107(01)00635-3

[12] A. Gokarna, S. V. Bhoraskar, N. R. Pavaskar and S. D. Sathaye, "Optoelectronic Characterization of Porous Silicon/CdS and ZnS Systems," Physica Status Solidi (A), Vol. 182, No. 1, 2000, pp. 175-179. doi:10.1002/1521-396X(200011)182:1<175::AID-PSSA1 75>3.0.CO;2-O

[13] L. K. Pan, Y. K. Ee, C. Q. Sun, G. Q. Yu, Q. Y. Zhang and B. K. Tay, "Band-Gap Expansion, Core-Level Shift, and Dielectric Suppression of Porous Silicon Passivated by Plasma Fluorination," Journal of Vacuum Science \& Technology B, Vol. 22, No. 2, 2004, pp. 583-587. 
doi:10.1116/1.1651108

[14] T. Katsube, M. Hara and I. Serizawa, "MOS-Type Micro-Oxygen Sensor Using $\mathrm{LaF}_{3}$ Workable at Room Temperature," Japanese Journal of Applied Physics, Vol. 29, No. 8, 1990, pp. L1392-L395.
[15] M. C. Liu, C. C. Lee, M. Kaneko, K. Nakahira and Y. Takano, "Microstructure Related Properties of Lanthanum Fluoride Films Deposited by Molybdenum Boat Evaporation at 193 nm," Thin Solid Films, Vol. 492, No. 1-2, 2005, pp. 45-49. 